\title{
A robust forward kinematics analysis of 3-Rㅛ planar platforms
}

\author{
Nicolás Rojas and Federico Thomas \\ Institut de Robòtica i Informàtica Industrial (CSIC-UPC), Barcelona, Spain \\ e-mail: \{nrojas, fthomas\}@iri.upc.edu
}

\begin{abstract}
The standard forward kinematics analysis of 3-RPR planar parallel platforms boils down to computing the roots of a sextic polynomial. There are many different ways to obtain this polynomial but all of them include exceptions for which the formulation is not valid. Unfortunately, near these exceptions the corresponding polynomial exhibits numerical instabilities. In this paper, we provide a way around this inconvenience by translating the forward kinematics problem to be solved into an equivalent problem fully stated in terms of distances. Using constructive geometric arguments, an alternative sextic which is not linked to a particular reference frame - is straightforwardly obtained without the need of variable eliminations nor tangent-half-angle substitutions. The presented formulation is valid, without any modification, for any planar 3-RPR parallel platform, including the special architectures and configurations - which ultimately lead to numerical instabilities - that cannot be directly handled by previous formulations.
\end{abstract}

Key words: 3-RPR parallel robots, coordinate-free formulations, Cayley-Menger determinants, bilateration, numerical stability

\section{Introduction}

Much has been written about the 3-RPR planar parallel robot because of its practical interest, mechanical simplicity, and rich mathematical properties [1]. Such a robot consist of a moving platform connected to the ground through three revolute-prismatic-revolute kinematic chains. The prismatic joint of each chain is actuated and the forward kinematics problem consists in, given the prismatic joint lengths, calculating the Cartesian pose of the moving platform. This problem has at most 6 different solutions which are usually computed by solving a system of three non-linear equations in three unknowns $[2,3]$. The major step in this resolution process is to find a so-called univariate characteristic equation in $\theta$ (the orientation of the moving platform), i.e., to eliminate all other variables from the system until an equation is obtained that contains only $\theta$. Finally, a tangent-half-angle substitution is applied to translate sine and cosine functions of $\theta$ into rational polynomial 
expressions in a new variable $t=\tan (\theta / 2)$. J-P. Merlet was probably one of the first researchers to observe that the computation of the roots of this $6^{\text {th }}$ degree leads, in some cases, to numerical instabilities and he also observed that the numerical robustness is improved by using a higher order polynomial $[4]$.

The tangent-half-angle substitution poses two well-known problems. One results from the fact that $\tan (\theta / 2)$ is undefined for $\pm \pi$. Moreover, it can become difficult to reconstruct other roots, occurring in conjunction with the $\operatorname{root} \theta= \pm \pi[5]$. The other problem is the introduction of extraneous roots. Both problems are well known and can be handled but it complicates notably subsequent calculations [6]. The use of the kinematic mapping, as in [7], avoids this substitution but the problem with $\pm \pi$ turns still remains if the used homogeneous coordinates are normalized to obtain the sextic polynomial. Other sources of numerical problems, specifically arising in 3RPR parallel robots, are discussed in [8].

In order to simplify as much as possible the coefficients of the resulting $6^{\text {th }}$-degree polynomial, it is possible to express the coordinates of the base attachments according to a specific coordinate frame. For example, by making one coordinate axis to coincide with the baseline between two base attachments and/or locating the origin at one base attachment. Nevertheless, this kind of simplifications has an important drawback: the numerical conditioning of the resulting formulation depends on the chosen reference frame. This is why those formulations which are not linked to a particular reference frame - or coordinate-free formulations - are preferable.

In this paper, we provide a way around the above difficulties by casting the problem into an equivalent problem fully stated in terms of distances which is solved by a sequence of bilaterations. As a result, a $6^{\text {th }}$-degree characteristic polynomial, which is not linked to any particular reference frame, is straightforwardly obtained without variable eliminations nor tangent-halfangle substitutions. Moreover, the obtained polynomial is mathematically more tractable than the one obtained using other approaches because its coefficients are the result of operating with Cayley-Menger determinants with geometric meaning.

This paper is organized as follows. A coordinate-free formula for bilateration expressed in terms of Cayley-Menger determinants is presented in Section 2. It is the basic formula, used in Section 3, to derive a coordinatefree characteristic polynomial for the general 3-RPR planar parallel robot. Section 4 analyzes a numerical example in which the standard formulations miss a solution. Finally, Section 5 summarizes the main points and gives prospects for further research. 


\section{Cayley-Menger determinants and bilateration}

Let $P_{i}$ and $\mathbf{p}_{i}$ denote a point and its position vector in a given reference frame, respectively. Then, let us define

$$
D\left(i_{1}, \ldots, i_{n} ; j_{1}, \ldots, j_{n}\right)=\left|\begin{array}{cccc}
0 & 1 & \ldots & 1 \\
1 & s_{i_{1}, j_{1}} & \ldots & s_{i_{1}, j_{n}} \\
\vdots & \vdots & \ddots & \vdots \\
1 & s_{i_{n}, j_{1}} & \ldots & s_{i_{n}, j_{n}}
\end{array}\right|
$$

with $s_{i, j}=\left\|\mathbf{p}_{i}-\mathbf{p}_{j}\right\|^{2}$, which is independent from the chosen reference frame. This determinant is known as the Cayley-Menger bi-determinant of the point sequences $P_{i_{1}}, \ldots, P_{i_{n}}$, and $P_{j_{1}}, \ldots, P_{j_{n}}$. When the two point sequences are the same, it will be convenient to abbreviate $D\left(i_{1}, \ldots, i_{n} ; i_{1}, \ldots, i_{n}\right)$ by $D\left(i_{1}, \ldots, i_{n}\right)$, which is simply called the Cayley-Menger determinant of the involved points.

In terms of Cayley-Menger determinants, the squared distance between $P_{i}$ and $P_{j}$ can be expressed as $D(i, j)$ and the signed area ${ }^{1}$ of the triangle $P_{i} P_{j} P_{k}$, as $\pm \frac{1}{2} \sqrt{D(i, j, k)}$. For a brief review of the properties of Cayley-Menger determinants, see [9].

The bilateration problem in $\mathbb{R}^{2}$ consists of finding the feasible locations of a point, say $P_{3}$, given its distances to two other points, say $P_{1}$ and $P_{2}$, whose locations are known.

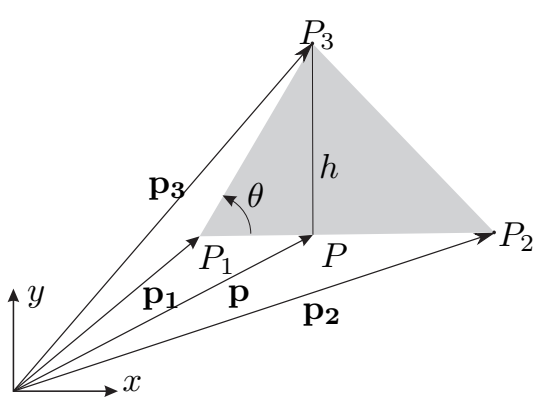

Fig. 1 The bilateration problem in $\mathbb{R}^{2}$ Then, according to Fig. 1, the position vector of the orthogonal projection of $P_{3}$ onto the line $P_{1} P_{2}$ can be expressed as:

$$
\mathbf{p}=\mathbf{p}_{1}+\sqrt{\frac{D(1,3)}{D(1,2)}} \cos \theta\left(\mathbf{p}_{2}-\mathbf{p}_{1}\right)=\mathbf{p}_{1}+\frac{D(1,2 ; 1,3)}{D(1,2)}\left(\mathbf{p}_{2}-\mathbf{p}_{1}\right) .
$$

Moreover, the position vector of $P_{3}$ can be expressed as:

$$
\mathbf{p}_{3}=\mathbf{p} \pm \frac{\sqrt{D(1,2,3)}}{D(1,2)} \mathbf{S}\left(\mathbf{p}_{2}-\mathbf{p}_{1}\right)
$$

\footnotetext{
${ }^{1}$ For a triangle $P_{i} P_{j} P_{k}$ in the Euclidean plane with area $A$, the signed area is defined as $+A$ (respectively, $-A$ ) if the point $P_{j}$ is to the right (respectively to the left) of the line $P_{i} P_{k}$, when going from $P_{i}$ to $P_{k}$
} 
where the \pm sign accounts for the two mirror symmetric locations of $P_{3}$ with respect to the line defined by $P_{1} P_{2}$, and $\mathbf{S}=\left[\begin{array}{cc}0 & -1 \\ 1 & 0\end{array}\right]$. Then, substituting (2) in (3) and expressing the result in matrix form, we obtain

$$
\mathbf{p}_{3}=\mathbf{p}_{1}+\mathbf{Z}\left(\mathbf{p}_{2}-\mathbf{p}_{1}\right)
$$

where

$$
\mathbf{Z}=\frac{1}{D(1,2)}\left[\begin{array}{cc}
D(1,2 ; 1,3) & \mp \sqrt{D(1,2,3)} \\
\pm \sqrt{D(1,2,3)} & D(1,2 ; 1,3)
\end{array}\right]
$$

\section{Distance-Based Coordinate-Free Formulation}

Fig. 2 shows a general 3-R $\underline{P R}$ planar parallel robot. The center of the three grounded passive revolute joints define the base triangle $P_{1} P_{2} P_{3}$ and the three moving passive revolute joints centers, the moving triangle $P_{4} P_{5} P_{6}$. The active prismatic joint variables are the lengths $\rho_{1}, \rho_{2}$, and $\rho_{3}$.

Next, we derive a coordinate-free formula for the forward kinematics of this parallel platform. To this end, instead of directly computing the Cartesian pose of the moving platform, first we will compute the set of values of $T=\left\|\mathbf{p}_{4}-\mathbf{p}_{2}\right\|^{2}$ compatible with $\rho_{1}, \rho_{2}$, and $\rho_{3}$ and the base and the moving platform side lengths, $l_{1}, l_{2}, l_{3}, L_{1}, L_{2}$, and $L_{3}$. Thus, this step is enterally posed in

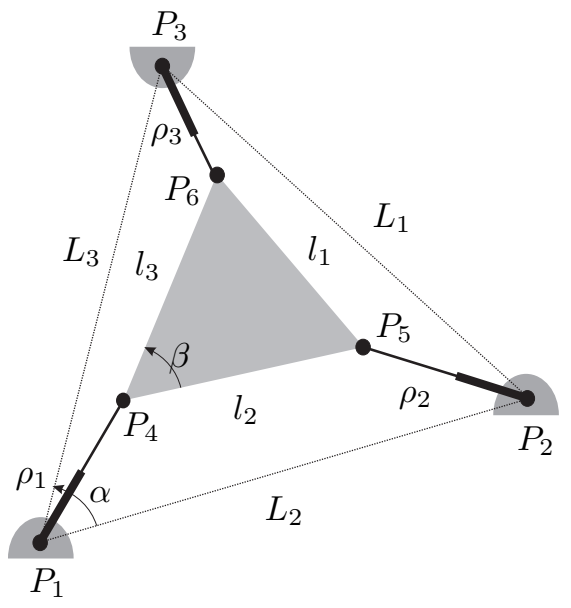

Fig. 2 A general planar 3-R $\underline{P R}$ parallel platform terms of distances.

Let us take $P_{1}$ and $P_{2}$ as reference points. Then, by bilateration

1. $\mathbf{p}_{3}$ can be computed from $\mathbf{p}_{1}$ and $\mathbf{p}_{2}$. This has only one solution because the oriented area of triangle $P_{1} P_{2} P_{3}$ is constant and its sign is determined by the sign of $\alpha$.

2. $\mathbf{p}_{4}$ can be computed from $\mathbf{p}_{1}$ and $\mathbf{p}_{2}$. This has two possible solutions which involve the unknown squared distance $T$.

3. $\mathbf{p}_{5}$ can be computed from $\mathbf{p}_{4}$ and $\mathbf{p}_{2}$. This also has two possible solutions which also involve the unknown squared distance $T$. 
4. $\mathbf{p}_{6}$ can be computed from $\mathbf{p}_{4}$ and $\mathbf{p}_{5}$. This has only one solution because the oriented area of triangle $P_{4} P_{5} P_{6}$ is constant and its sign is determined by the sign of $\beta$.

Translating the above four bilaterations into algebraic terms using (4), the following system of vector linear equations is obtained:

$$
\left.\begin{array}{l}
\mathbf{p}_{3}=\mathbf{p}_{1}+\mathbf{A}\left(\mathbf{p}_{2}-\mathbf{p}_{1}\right) \\
\mathbf{p}_{4}=\mathbf{p}_{1}+\mathbf{B}\left(\mathbf{p}_{2}-\mathbf{p}_{1}\right) \\
\mathbf{p}_{5}=\mathbf{p}_{4}+\mathbf{C}\left(\mathbf{p}_{2}-\mathbf{p}_{4}\right) \\
\mathbf{p}_{6}=\mathbf{p}_{5}+\mathbf{D}\left(\mathbf{p}_{4}-\mathbf{p}_{5}\right)
\end{array}\right\}
$$

where

$$
\begin{aligned}
& \mathbf{A}=\frac{1}{D(1,2)}\left[\begin{array}{cc}
D(1,2 ; 1,3) & -\operatorname{sign}(\alpha) \sqrt{D(1,2,3)} \\
\operatorname{sign}(\alpha) \sqrt{D(1,2,3)} & D(1,2 ; 1,3)
\end{array}\right] \\
& \mathbf{B}=\frac{1}{D(1,2)}\left[\begin{array}{cc}
D(1,2 ; 1,4) & \mp \sqrt{D(1,2,4)} \\
\pm \sqrt{D(1,2,4)} & D(1,2 ; 1,4)
\end{array}\right] \\
& \mathbf{C}=\frac{1}{D(4,2)}\left[\begin{array}{cc}
D(4,2 ; 4,5) & \mp \sqrt{D(4,2,5)} \\
\pm \sqrt{D(4,2,5)} & D(4,2 ; 4,5)
\end{array}\right] \\
& \mathbf{D}=\frac{1}{D(5,4)}\left[\begin{array}{cc}
D(5,4 ; 5,6) & \operatorname{sign}(\beta) \sqrt{D(5,4,6)} \\
-\operatorname{sign}(\beta) \sqrt{D(5,4,6)} & D(5,4 ; 5,6)
\end{array}\right] .
\end{aligned}
$$

Now, by properly manipulating the equations in the above linear system, it is possible to conclude that

$$
\left(\mathbf{p}_{6}-\mathbf{p}_{3}\right)=\boldsymbol{\Omega}\left(\mathbf{p}_{\mathbf{2}}-\mathbf{p}_{\mathbf{1}}\right)
$$

where $\boldsymbol{\Omega}=-\mathbf{A}+\mathbf{B}+\mathbf{C}-\mathbf{D C}-\mathbf{C B}+\mathbf{D C B}$. This matrix, when expanded in terms of Cayley-Menger determinants, leads to:

$$
\boldsymbol{\Omega}=\frac{1}{D(5,4) D(4,2) D(1,2)}\left[\begin{array}{cc}
w_{1} & -w_{2} \\
w_{2} & w_{1}
\end{array}\right]
$$

where

$$
\begin{aligned}
w_{1}= & D(4,6 ; 4,5)( \pm \sqrt{D(1,2,4)})( \pm \sqrt{D(4,2,5)}) \\
& +D(4,2 ; 4,5)(\operatorname{sign}(\beta) \sqrt{D(5,4,6)})( \pm \sqrt{D(1,2,4)}) \\
& -D(2,4 ; 2,1)(\operatorname{sign}(\beta) \sqrt{D(5,4,6)})( \pm \sqrt{D(4,2,5)}) \\
& +D(4,6 ; 4,5) D(2,4 ; 2,1) D(4,2 ; 4,5) \\
& +D(5,4) D(4,2)(D(1,2 ; 1,4)-D(1,2 ; 1,3))
\end{aligned}
$$

and 


$$
\begin{aligned}
w_{2}= & (\operatorname{sign}(\beta) \sqrt{D(5,4,6)})( \pm \sqrt{D(1,2,4)})( \pm \sqrt{D(4,2,5)}) \\
& +D(4,6 ; 4,5) D(2,4 ; 2,1)( \pm \sqrt{D(4,2,5)}) \\
& +D(4,2 ; 4,5) D(2,4 ; 2,1)(\operatorname{sign}(\beta) \sqrt{D(5,4,6)}) \\
& -D(5,4) D(4,2)(\operatorname{sign}(\alpha) \sqrt{D(1,2,3)}) \\
& +D(5,4) D(4,2)( \pm \sqrt{D(1,2,4)}) \\
& -D(4,2 ; 4,5) D(4,6 ; 4,5)( \pm \sqrt{D(1,2,4)})
\end{aligned}
$$

Now, it can be checked that

$$
\frac{\left\|\mathbf{p}_{6}-\mathbf{p}_{3}\right\|^{2}}{\left\|\mathbf{p}_{2}-\mathbf{p}_{1}\right\|^{2}}=\operatorname{det}(\boldsymbol{\Omega})
$$

Thus,

$$
\frac{D(6,3)}{D(1,2)}=\frac{w_{1}^{2}+w_{2}^{2}}{D^{2}(5,4) D^{2}(4,2) D^{2}(1,2)},
$$

which is a scalar equation in one unknown: $T$. Finally, by expanding all the involved Cayley-Menger determinants in the above equation in terms of distances, we obtain

$$
\Phi_{a}+\Phi_{b} A_{124}+\Phi_{c} A_{425}+\Phi_{d} A_{124} A_{425}=0
$$

where

$$
A_{124}= \pm \frac{1}{2} \sqrt{\left(\left(L_{2}+\rho_{1}\right)^{2}-T\right)\left(T-\left(L_{2}-\rho_{1}\right)^{2}\right)}
$$

and

$$
A_{425}= \pm \frac{1}{2} \sqrt{\left(\left(l_{2}+\rho_{2}\right)^{2}-T\right)\left(T-\left(l_{2}-\rho_{2}\right)^{2}\right)}
$$

are the signed areas of the triangles $P_{1} P_{2} P_{4}$ and $P_{4} P_{2} P_{5}$, respectively. $\Phi_{a}$, $\Phi_{b}, \Phi_{c}$, and $\Phi_{d}$ are polynomials in $T$ whose expressions can be found in the appendix.

By properly twice squaring equation (9) to eliminate the two squared roots involving $T$, a $6^{\text {th }}$-degree polynomial in $T$ is finally obtained. The roots of this polynomial determine the assembly-modes of the analyzed robot. Given the position vectors $\mathbf{p}_{1}$ and $\mathbf{p}_{2}$ in a particular reference frame, each root of this polynomial determines up to four possible values for $\mathbf{p}_{6}$ that can be obtained through a sequence of bilaterations. At least one of the values obtained from each of these sequences must satisfy, by construction, the distance constraint between $P_{3}$ and $P_{6}$ (i.e., $\left.D(3,6)=\rho_{3}^{2}\right)$. Observe that the sequence of bilat- 
erations is not the same depending on whether the root is at the origin or not. Actually, it is important to realize that the obtained sextic permits to compute the solutions in which $T=0$.

The presented formulation is coordinate-free because it is independent from the chosen reference frame. It is valid, without any modifications, for any planar 3-RPR parallel platform, including the special architectures discussed in [3] and [8] that cannot be directly solved by the formulation presented in [2], or that have been solved on an ad hoc basis [10].

\section{Numerical Example}

Let us study the planar 3 -R $\underline{P R}$ parallel platform defined by $l_{1}^{2}=25, l_{2}^{2}=36$, $l_{3}^{2}=25, L_{1}^{2}=73, L_{2}^{2}=16, L_{3}^{2}=65, \alpha>0$, and $\beta>0$, with input variables $\rho_{1}^{2}=1, \rho_{2}^{2}=121$, and $\rho_{3}^{2}=169$. If $\mathbf{p}_{1}=(0,0)^{T}, \mathbf{p}_{2}=(4,0)^{T}$, and $\mathbf{p}_{3}=(1,8)^{T}$, it can be verified that the characteristic polynomial of this mechanism, using the formulation derived in [2], reduces to:

$$
1469440 \Gamma^{4}+1755136 \Gamma^{3}+4261376 \Gamma^{2}+1140736 \Gamma+219136=0
$$

with $\sin (\theta)=\frac{2 \Gamma}{1+\Gamma^{2}}$ and $\cos (\theta)=\frac{1-\Gamma^{2}}{1+\Gamma^{2}}, \theta$ being the angle between the lines defined by $P_{1} P_{2}$ and $P_{4} P_{5}$. The solutions of equation (10), obtained using 32 decimal digits in the computations, are $-0.4573-1.5419 i,-0.4573+1.5419 i$, $-0.1399-0.1952 i$, and $-0.1399+0.1952 i$. Since none of them is real, it can be erroneously concluded that the platform under study cannot be assembled with the given leg lengths.

Alternately, using the formulation derived in [10], the following characteristic polynomial is obtained:

$$
4408320 Y^{4}-1744896 Y^{3}+7788032 Y^{2}-1464320 Y+3564544=0
$$

where $\sin (\psi)=\frac{2 Y}{1+Y^{2}}$ and $\cos (\psi)=\frac{1-Y^{2}}{1+Y^{2}}, \psi$ being the angle between the lines defined by $P_{1} P_{4}$ and $P_{1} P_{2}$. The solutions of this equation obtained using 32 decimal digits in the computations, are $-0.0363-0.9243 i,-0.0363+0.9243 i$, $0.2342-0.9435 i$, and $0.2342+0.9435 i$. Again, since none of them is real, it can be erroneously concluded that the platform under study cannot be assembled with the given leg lengths thus confirming the results obtained using the formulation proposed in [2]. The formulation described in [7] leads to an analogous situation when one of the homogeneous coordinates is normalized to 1 . Using the implementation for this formulation reported in [12], and choosing the moving reference frame such that $\mathbf{p}_{4}=(0,0)^{T}$ and $\mathbf{p}_{5}=(6,0)^{T}$ in it, the resulting polynomial is: 
$1469440 x_{1}{ }^{4}+1755136 x_{1}{ }^{3}+4261376 x_{1}{ }^{2}+1140736 x_{1}+219136=0$

where $x_{1}$ is a component of the kinematic image space coordinates. The roots of this equation are $-0.4573-1.5419 i,-0.4573+1.5419 i,-0.1399-$ $0.1952 i$, and $-0.1399+0.1952 i$. Again, none of them is real. Nevertheless, substituting the parameters of the mechanism under study and the values of the input variables given above in the coordinate-free equation (9), the following characteristic polynomial is obtained:

$$
B_{6} T^{6}+B_{5} T^{5}+B_{4} T^{4}+B_{3} T^{3}+B_{2} T^{2}+B_{1} T^{1}+B_{0}=0
$$

where

$$
\begin{aligned}
& B_{6}=-3445000, B_{5}=-185454160, B_{4}=304547661 \\
& B_{3}=416912277408, B_{2}=-32392769185400 \\
& B_{1}=121165627563000, \text { and } B_{0}=-15097679553125000 .
\end{aligned}
$$

The roots of this equation, calculated with the same numerical precision as above, are $-65.0725-$ $41.0946 i,-65.0725+41.0946 i, 13.1561$ $-31.7921 i, 13.1561+31.7921 i$, and a double root at 25.0000 . It can be checked that the obtained double real root corresponds to a valid configuration of the analyzed 3-RPR parallel platform, in clear contradiction with what was concluded using the formulations proposed in [2], [10], and [12]. In the platform pose associated with this double root, $\theta=0, \psi=\pi$, and $\mathbf{p}_{4}=(-1,0)^{T}$. Fig. 3 depicts this configuration.

The obtained results confirm that the formulation of Gosselin et. al. [2] and that of Kong and Gosselin [10]

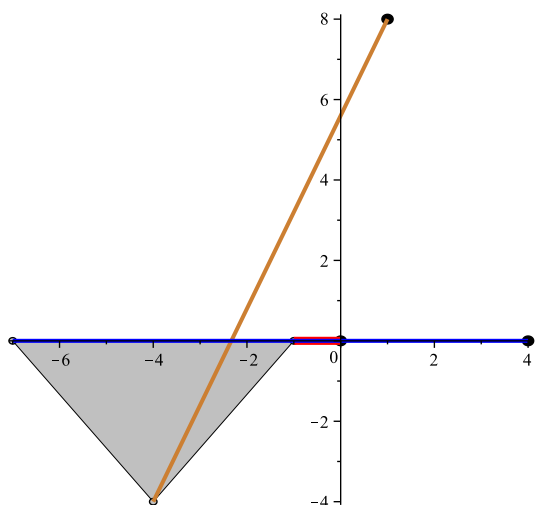

Fig. 3 This solution for the analyzed example cannot be obtained using the formulations presented in [2], [10], and [12]. are not, in general, robust. Moreover, depending on the location of the chosen reference frames, the formulation of Husty [7] would also fail to provide all solutions. This is a highly relevant fact for the kinematic analysis and non-singular assembly-mode change studies of 3-R $\underline{P R}$ parallel manipulators [7], [11]. The presented coordinate-free formulation does not exhibit this kind of undesirable behavior. 


\section{Conclusions}

Stating the forward kinematics analysis of 3-RPR parallel platforms directly in terms of poses introduces two major disadvantages: (a) a reference frame has to be introduced, and (b) all formulas involve translations and rotations simultaneously. This paper proposes a different approach in which, instead of directly computing the sought Cartesian poses, a problem fully posed in terms of distances is first solved. Then, the original problem can be trivially solved by sequences of bilaterations.

All proposed previous formulations have exceptions which translate into numerical instabilities when close to them. The approach proposed in this paper has no exceptions and, since all coefficients involved in the associated formulation are the result of operating with Cayley-Menger determinants with geometric meaning, it opens the door to a rational and exhaustive classification of all 3-RPR planar platforms whose characteristic polynomials can be simplified either because they can be factorized or because their degrees are lower than six. This is certainly a point that deserves further attention.

Acknowledgements We are very much indebted to Philippe Wenger and his colleagues from IRCCyN who inspired this work. We also gratefully acknowledge the financial support of the Autonomous Government of Catalonia through the VALTEC programme, cofinanced with FEDER funds, and the Colombian Ministry of Communications and Colfuturo through the ICT National Plan of Colombia.

\section{References}

1. Merlet, J-P.: Parallel robots, Kluwer academic publishers, Dorchecht, The Netherlands (2000)

2. Gosselin, C., Sefrioui, J., and Richard, M.J.: Solution polynomiale au problème de la cinématique directe des manipulateurs parallèles plans à 3 degrés de liberté. Mechanism and Machine Theory, 27(2):107-119 (1992)

3. Gosselin, C., and Merlet, J-P.: On the direct kinematics of planar parallel manipulators: special architectures and number of solutions. Mechanism and Machine Theory, 29(8):1083-1097 (1994)

4. Mertlet, J-P.: 5BARRES user's manual V. 0.1, available at http://wwwsop.inria.fr/coprin/logiciels/RP/FK/3-RPR/notice-html.html

5. Lipkin, H., and Duffy, J.: A vector analysis of robot manipulators. In: Recent advances in robotics (Eds. Beni, G., Hackwood, S.J.), Wiley, New York, 175-242 (1985)

6. Kovács, P., and Hommel, G.: On the tangent-half-angle substitution. In: Computational Kinematics (Eds. Angeles, J., Hommenl, G., and Kovács, P.), Kluwer Academic Publishers, Dordrecht, 27-39 (1993)

7. Husty, M.: Non-singular assembly mode change in 3-RPR-parallel manipulators. In: Computational Kinematics (Eds. Kecskeméthy, A., Müller, A.), Springer, Berlin, Heidelberg, 51-60 (2009)

8. Wenger, P., Chablat, D., and Zein, M.: Degeneracy study of the forward kinematics of planar 3-RPR parallel manipulators. ASME Journal of Mechanical Design, 192(12):1265-1268 (2007) 
9. Thomas, F., and Ros, L.: Revisiting trilateration for robot localization. IEEE Trans. on Robotics, 21(1):93-101 (2005)

10. Kong, X., and Gosselin, C.: Forward displacement analysis of third-class analytic 3RPR planar parallel manipulators. Mechanism and Machine Theory, 39(9):1009-1018 (2001)

11. Zein, M., Wenger, P., and Chablat, D.: Non-singular assembly-mode changing motions for 3-RPR parallel manipulators. Mechanism and Machine Theory, 43:480-490 (2008)

12. Hayes, M. J. D.: Kinematics of General Planar Stewart-Gough Platforms. PhD Thesis, Department of Mechanical Engineering and Centre for Intelligent Machines, McGill University (1999)

\section{Appendix}

The polynomials $\Phi_{a}, \Phi_{b}, \Phi_{c}$, and $\Phi_{d}$ in equation (9) can be expressed as:

$$
\Phi_{a}=a T^{2}+b T+c, \Phi_{b}=d T+e, \Phi_{c}=f T+g, \text { and } \Phi_{d}=h
$$

where

$$
\begin{aligned}
a= & -l_{3}^{2} L_{1}^{2}+3 l_{3}^{2} L_{2}^{2}-3 l_{1}^{2} L_{2}^{2}-l_{1}^{2} L_{3}^{2}+l_{3}^{2} L_{3}^{2}+l_{1}^{2} L_{1}^{2}-3 l_{2}^{2} L_{3}^{2}-l_{2}^{2} L_{2}^{2}+3 l_{2}^{2} L_{1}^{2} \\
& +\operatorname{sign}(\alpha) \operatorname{sign}(\beta) A_{123} A_{546} \\
b= & -3 l_{2}^{2} \rho_{1}^{2} L_{2}^{2}+l_{2}^{4} L_{3}^{2}-5 L_{2}^{2} l_{2}^{2} l_{3}^{2}+l_{2}^{2} \rho_{2}^{2} L_{1}^{2}-3 L_{2}^{2} l_{3}^{2} \rho_{2}^{2}+3 L_{2}^{2} l_{1}^{2} \rho_{2}^{2} \\
& -3 l_{2}^{2} L_{2}^{2} \rho_{2}^{2}-\rho_{1}^{2} L_{2}^{2} l_{1}^{2}-l_{2}^{4} L_{1}^{2}+3 l_{2}^{4} L_{2}^{2}-5 l_{2}^{2} L_{2}^{2} L_{1}^{2}-3 l_{2}^{2} L_{2}^{2} L_{3}^{2} \\
& +L_{2}^{2} l_{1}^{2} L_{1}^{2}-L_{2}^{2} l_{1}^{2} L_{3}^{2}+l_{1}^{2} l_{2}^{2} L_{1}^{2}-l_{1}^{2} l_{2}^{2} L_{3}^{2}-L_{2}^{4} l_{3}^{2}+3 l_{2}^{2} \rho_{1}^{2} L_{3}^{2}+l_{1}^{2} \rho_{2}^{2} L_{3}^{2} \\
& -3 L_{2}^{2} l_{1}^{2} l_{2}^{2}-L_{2}^{2} l_{3}^{2} L_{1}^{2}+L_{2}^{2} l_{3}^{2} L_{3}^{2}-l_{2}^{2} l_{3}^{2} L_{1}^{2}+l_{2}^{2} l_{3}^{2} L_{3}^{2}+8 l_{2}^{2} L_{2}^{2} \rho_{3}^{2} \\
& +L_{2}^{4} l_{1}^{2}-3 l_{2}^{2} \rho_{1}^{2} L_{1}^{2}+l_{3}^{2} \rho_{1}^{2} L_{1}^{2}-l_{2}^{2} \rho_{2}^{2} L_{3}^{2}+\rho_{1}^{2} L_{2}^{2} l_{3}^{2}-l_{1}^{2} \rho_{1}^{2} L_{1}^{2}-l_{1}^{2} \rho_{2}^{2} L_{1}^{2} \\
& +l_{1}^{2} \rho_{1}^{2} L_{3}^{2}-l_{3}^{2} \rho_{1}^{2} L_{3}^{2}+3 l_{2}^{2} L_{2}^{4}+l_{3}^{2} \rho_{2}^{2} L_{1}^{2}-l_{3}^{2} \rho_{2}^{2} L_{3}^{2} \\
& +\left(-\rho_{2}^{2}+l_{2}^{2}+L_{2}^{2}-\rho_{1}^{2}\right) \operatorname{sign}(\alpha) \operatorname{sign}(\beta) A_{123} A_{546} \\
c= & -\left(l_{2}-\rho_{2}\right)\left(l_{2}+\rho_{2}\right)\left(L_{2}-\rho_{1}\right)\left(L_{2}+\rho_{1}\right) \\
& {\left[-\left(l_{2}^{2}+l_{3}^{2}-l_{1}^{2}\right)\left(L_{1}^{2}+L_{2}^{2}-L_{3}^{2}\right)+\operatorname{sign}(\alpha) \operatorname{sign}(\beta) A_{1,2,3} A_{5,4,6}\right] } \\
d=2 & \left(-l_{3}^{2}+l_{1}^{2}+3 l_{2}^{2}\right) \operatorname{sign}(\alpha) A_{123}+2\left(-L_{2}^{2}-L_{1}^{2}+L_{3}^{2}\right) \operatorname{sign}(\beta) A_{546} \\
e=2 & \left(l_{2}-\rho_{2}\right)\left(l_{2}+\rho_{2}\right) \\
& {\left[\left(-l_{2}^{2} l_{3}^{2}+l_{1}^{2}\right) \operatorname{sign}(\alpha) A_{123}+\left(-L_{2}^{2}-L_{1}^{2}+L_{3}^{2}\right) \operatorname{sign}(\beta) A_{546}\right] } \\
f=2 & \left(-l_{3}^{2}+l_{1}^{2}-l_{2}^{2}\right) \operatorname{sign}(\alpha) A_{123}+2\left(3 L_{2}^{2}-L_{1}^{2}+L_{3}^{2}\right) \operatorname{sign}(\beta) A_{546} \\
g=2 & \left(L_{2}-\rho_{1}\right)\left(L_{2}+\rho_{1}\right) \\
& {\left[\left(-l_{2}^{2} l_{3}^{2}+l_{1}^{2}\right) \operatorname{sign}(\alpha) A_{123}+\left(-L_{2}^{2}-L_{1}^{2}+L_{3}^{2}\right) \operatorname{sign}(\beta) A_{546}\right] } \\
h= & \left(l_{2}^{2}+l_{3}^{2}-l_{1}^{2}\right)\left(L_{1}^{2}+L_{2}^{2}-L_{3}^{2}\right)-4 \operatorname{sign}(\alpha) \operatorname{sign}(\beta) A_{123} A_{546} \\
&
\end{aligned}
$$

